\title{
A New Objective Parameter in Hydroxychloroquine-Induced Retinal Toxicity Screening Test: Macular Retinal Ganglion Cell-Inner Plexiform Layer Thickness
}

\author{
Mehmet BULUT, ${ }^{1}$ Muhammet Kazım EROL, ${ }^{1}$ Devrim TOSLAK, ${ }^{1}$ \\ Melih AKIDAN, ${ }^{1}$ Ebru KAYA BAŞAR, ${ }^{2}$ Hasan Fatih ÇAY ${ }^{3}$ \\ ${ }^{1}$ Department of Ophthalmology, Antalya Training and Research Hospital, Antalya, Turkey \\ ${ }^{2}$ Deparment of Animal Science Biometry and Genetics Unit, Akdeniz University, Faculty of Agriculture, Antalya, Turkey \\ ${ }^{3}$ Department of Rheumatology, Antalya Education and Research Hospital, Physical Medicine Rehabilitation, Antalya, Turkey
}

\begin{abstract}
Objectives: This study aims to detect hydroxychloroquine (HCQ)-induced retinal toxicity at an earlier stage through the use of spectral-domain optical coherence tomography device, especially by measuring macular retinal ganglion cell-inner plexiform layer (RGC-IPL) thickness.

Patients and methods: In this study, 92 eyes of 46 Caucasian female patients (mean age $53.6 \pm 8.1$ years; range 32 to 69 years) who were taking HCQ were assigned to group 1, while 80 eyes of 40 age-matched Caucasian female control subjects (mean age $56.1 \pm 10.7$ years; range 34 to 71 years) were assigned to group 2. RGC-IPL thickness and retinal nerve fiber layer thickness were measured in all subjects by Cirrus high-definition optical coherence tomography model 5000 device using macular cube $512 \times 128$ and optic disc cube $200 \times 200$ protocols. We performed an evaluation to see if there was any difference between the measured values of the groups. The correlation between average RGC-IPL thickness measures and cumulative dose of $\mathrm{HCQ}$ and duration of use was analyzed.

Results: Retinal ganglion cell-inner plexiform layer of group 1 was found to be statistically thinner than that of group 2 both on average and in all segments (superior, superonasal, inferonasal, inferotemporal and superotemporal) except inferior segment when segmented ( $\mathrm{p}<0.05)$. Additionally, a statistically significant negative correlation was found between the average RGC-IPL thickness and cumulative dose of HCQ ( $r=-0.371, p=0.001)$ as well as the duration of use $(r=-0.308, p=0.006)$.

Conclusion: Patients taking HCQ were found to have decreased RGC-IPL thickness at an early stage due to retinal toxicity induced by the drug. We think that measuring the RGC-IPL thickness may become an important objective in HCQ screening tests.

Keywords: Hydroxychloroquine; macula; perimetry; retinal ganglion cell; spectral-domain optical coherence tomography.
\end{abstract}

Chloroquine (CQ) and its chemical analogue hydroxychloroquine (HCQ) -known as antimalarial drugs- have been used commonly since 1950s for the treatment of various autoimmune rheumatic diseases. ${ }^{1}$ The efficacy and toxicity of HCQ are lower than those of CQ. ${ }^{2}$ Ocular toxicity induced by these drugs was first described in $1957 .^{3}$ These drugs are eliminated from the body very slowly. Their deposits on cornea, ciliary body, lens, retina and choroid may lead to ocular side effects which are usually reversible upon discontinuation of drug. ${ }^{1}$ The most common ocular toxicity induced by antimalarials presents as bull's eye maculopathy. ${ }^{4}$ When retinal toxicity develops, it leads to irreversible and sometimes progressive loss of visual acuity, color vision, and central visual field defects. ${ }^{5}$ It is important to detect retinal toxicity before it leads to irreversible consequences. ${ }^{6}$ There are several tests that are used to detect retinal toxicity such as visual field analysis, fundus autofluorescence photography and electroretinography (ERG).? 
Previous histopathological studies conducted on the retinas of people with $\mathrm{CQ}$ retinopathy demonstrated that photoreceptors and neural retina were damaged. ${ }^{8,9}$ In their study on monkeys, Rosenthal et al. ${ }^{10}$ showed that CQ resulted in the degeneration of retinal ganglion cells. Pasadhika et al. ${ }^{11}$ showed that perifoveal retinal ganglion cell-inner plexiform layer (RGC-IPL) thickness decreased in patients who had been taking HCQ for a long time before any functional or structural impairment occurred in retinal pigment epithelium layer and photoreceptors. The number of patients in that study, however, was very low and they did not examine the correlation between perifoveal thinning and use of HCQ.

In recent years, spectral domain-optical coherence tomography (SD-OCT) has been used commonly for early diagnosis of retinal impairment. Macular RGC-IPL thickness measured by SD-OCT can be used to evaluate the structural changes in RGCs. Therefore, in this study, we aimed to detect HCQ-induced retinal toxicity at an earlier stage through the use of SD-OCT device, especially by measuring macular RGC-IPL thickness.

\section{PATIENTS AND METHODS}

Patients who were using HCQ for the treatment of rheumatic diseases such as rheumatoid arthritis, Sjögren's syndrome, or connective tissue diseases were enrolled in this study, which was conducted at Antalya Training and Research Hospital between April 2016 and August 2016. A total of 92 eyes of 46 Caucasian female patients (mean age $53.6 \pm 8.1$ years; range 32 to 69 years) who were taking HCQ were assigned to group 1, while 80 eyes of 40 age-matched Caucasian female control subjects (mean age $56.1 \pm 10.7$ years; range 34 to 71 years) were assigned to group 2 . The study protocol was approved by the Antalya Training and Research Hospital Ethics Committee. A written informed consent was obtained from each patient. The study was conducted in accordance with the principles of the Declaration of Helsinki.

The inclusion criteria for the study were as follows: patients older than 18 years, having a refractive error less than \pm 5 diopter, capable of undergoing visual field testing and having a signal amplitude in SD-OCT measurement of higher than $6 / 10$. The exclusion criteria were: previous treatment with $\mathrm{CQ}$, presence of disorders which might affect ocular structures such as diabetes mellitus, neurodegenerative diseases, thyroid dysfunction, glaucoma, macular diseases, uveitis or vascular diseases.

Age, sex, diagnosis, the daily dose of HCQ, cumulative dose of HCQ and duration of use were recorded for all patients. According to the ophthalmological examination protocol, all patients were subjected to visual acuity test using Snellen chart; ocular tension measurement using Goldmann applanation tonometer; biomicroscopic examination; central 10-degree visual field test using Octopus 900 (Interzeag AG, Schlieren-Zurich, Switzerland); fundus autofluorescence photography and color fundus photograph examination using Visucam NM/FA (Carl Zeiss, Germany), and macular RGC-IPL thickness and peripapillary retinal nerve fiber layer (RNFL) thickness measurements using Cirrus high-definition OCT, model 5000 (Carl Zeiss Meditec Inc., Jena, Germany). All OCT measurements and visual field tests were performed between $9 \mathrm{am}$ and 11 am on the same day. Both eyes of the subjects were studied.

All OCT measurements were performed by the same experienced technician. Before the measurements, the pupils of all patients' were dilated using 1\% tropicamide (Tropamid; Bilim, Istanbul, Turkey). Average peripapillary RNFL thickness was measured using optic disc cube $200 \times 200$ protocol. Measurement was applied on superior, nasal, inferior and temporal quadrants; then the arithmetic mean of these measurements was calculated and named average RNFL thickness. The average macular RGC-IPL thickness was measured using macular cube $512 \times 128$ protocol (Figure 1). Measurement was applied on superior, superonasal, inferonasal, inferotemporal and superotemporal sites; then the arithmetic mean of these measurements was calculated and named average RGC-IPL thickness. SD-OCT images with a strength quality less than 6/10 were excluded.

\section{Statistical analysis}

Data obtained from the study were analyzed using SPSS software. Levene's test was used to 

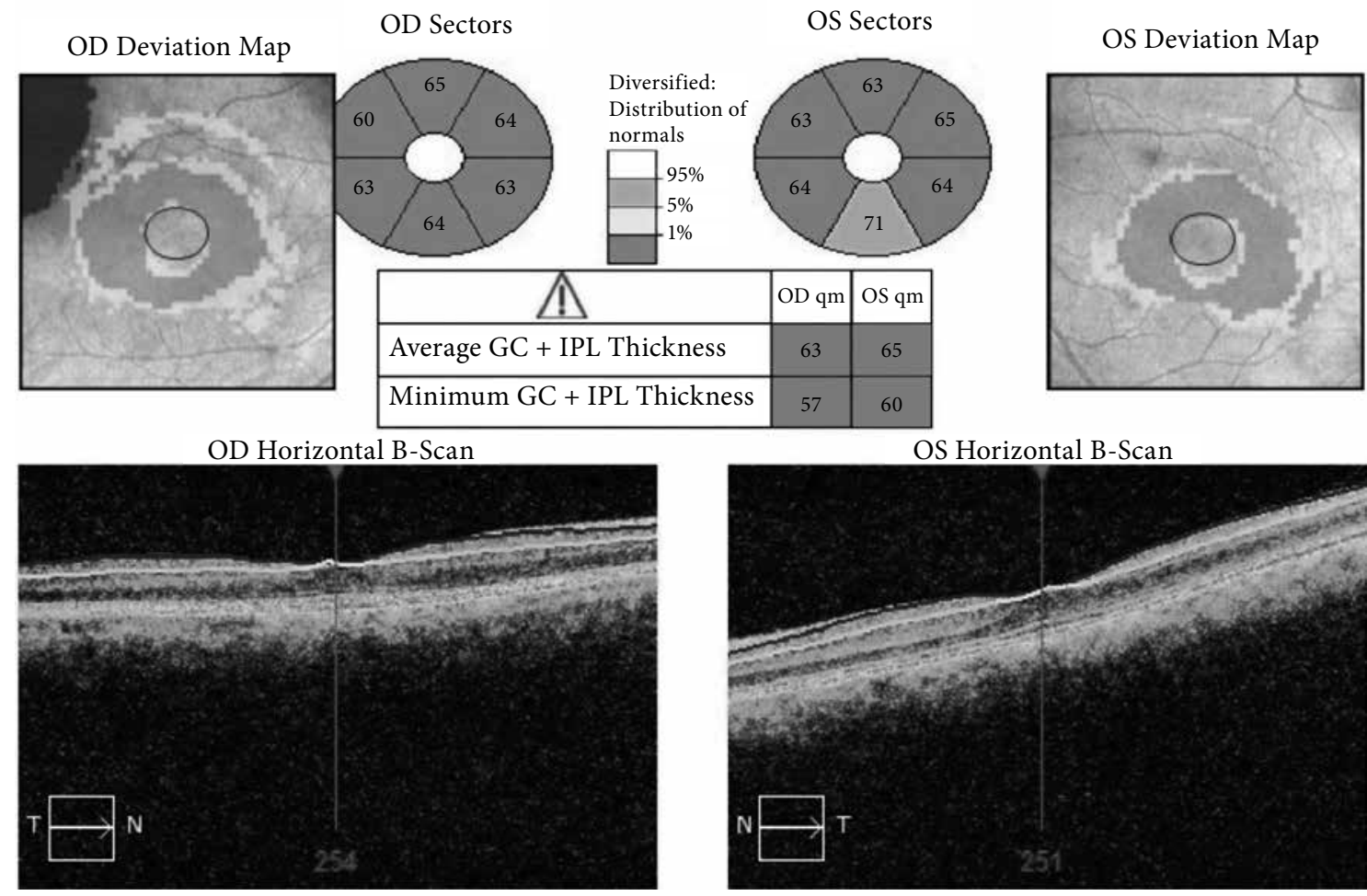

Figure 1. This figure shows reduced macular ganglion cell-inner plexiform layer (GC-IPL) thickness in both eyes with hydroxychloroquine (HCQ) retinopathy of a 54-year-old female patient who was diagnosed with systemic lupus erythematosus and had used HCQ for 58 months. A cumulative dose of HCQ was $696 \mathrm{~g}$ and daily dose was $400 \mathrm{mg} /$ day. OD: Oculus dextrus; OS: Oculus sinister.

assess the homogeneity of variances, which is a precondition for parametric tests, while ShapiroWilk test was used to check the assumption of normality. Independent $\mathrm{t}$-test was used to assess the differences between the two groups. Moreover, Pearson correlation coefficient was used to analyze the correlation between the continuous variables. $\mathrm{P}<0.05$ was considered to be statistically significant in all tests.

\section{RESULTS}

The mean age was statistically similar in both groups ( $\mathrm{p}=0.386$, Table 1$)$. The mean cumulative dose of HCQ was $543.6 \pm 340.8 \mathrm{~g}$, daily dose was $302.5 \pm 101.2 \mathrm{mg}$ and duration of treatment was $58.1 \pm 38.3$ months in group 1 .

The average RGC-IPL was found to be statistically thinner in group 1 . The segmental

Table 1. Demographic and clinical characteristics of study subjects

\begin{tabular}{|c|c|c|c|c|c|c|c|}
\hline & \multicolumn{3}{|c|}{ Group $1(n=46)$} & \multicolumn{3}{|c|}{ Group $2(n=40)$} & \multirow[b]{2}{*}{$p^{*}$} \\
\hline & $\mathrm{n}$ & $\%$ & Mean \pm SD & $\mathrm{n}$ & $\%$ & Mean \pm SD & \\
\hline Age (year) & & & $53.6 \pm 8.1$ & & & $56.1 \pm 10.7$ & 0.386 \\
\hline Cumulative drug dose (g) & & & $543.6 \pm 340.8$ & & & 0 & NA \\
\hline Duration (month) & & & $58.1 \pm 38.3$ & & & 0 & NA \\
\hline Daily dose (mg) & & & $302.5 \pm 101.2$ & & & 0 & NA \\
\hline \multicolumn{8}{|l|}{ Primary diagnosis } \\
\hline Rheumatoid arthritis & 12 & 26 & & 0 & 0 & & \\
\hline Systemic lupus erythematosus & 8 & 17.3 & & 0 & 0 & & \\
\hline Connective tissue disease & 7 & 15.2 & & 0 & 0 & & \\
\hline Sjögren's syndrome & 19 & 41.3 & & 0 & 0 & & \\
\hline
\end{tabular}




\begin{tabular}{|c|c|c|c|}
\hline & Group 1 ( $n=92$ eyes) & Group 2 ( $\mathrm{n}=80$ eyes) & \\
\hline & Mean \pm SD & Mean \pm SD & $p^{*}$ \\
\hline \multicolumn{4}{|c|}{ RGC-IPL thickness ( $\mu \mathrm{m})$} \\
\hline Average $e^{* * *}$ & $83.8 \pm 8.7$ & $86.5 \pm 5.5$ & 0.033 \\
\hline Superior & $84.9 \pm 10.6$ & $87.9 \pm 5.8$ & 0.032 \\
\hline Superio-nasal & $82.6 \pm 7.2$ & $85.1 \pm 6.1$ & 0.030 \\
\hline Inferio-nasal & $83.4 \pm 7.0$ & $85.6 \pm 5.8$ & 0.047 \\
\hline Inferior & $83.5 \pm 9.0$ & $85.3 \pm 5.8$ & 0.139 \\
\hline Inferio-temporal & $84 \pm 9.8$ & $86.6 \pm 6.1$ & 0.043 \\
\hline Superio-temporal & $84.9 \pm 11.8$ & $88.7 \pm 5.6$ & 0.010 \\
\hline
\end{tabular}

measurements of RGC-IPL thickness revealed that group 1 had statistically significant thinning in all their macular segments (superior, superonasal, inferonasal, inferotemporal and superotemporal) except inferior segment as compared to group 2 (Table 2).

The average peripapillary RNFL thickness and segmented (superior, nasal, inferior, temporal) RNFL measurements were statistically similar in both groups. Standard automated 10-2 perimetry performed using Octopus visual field testing device revealed that the mean defect (MD) was $2.7 \pm 1.6-2.1 \pm 1.8 \mathrm{db}$. and standard loss of variance (sLV) was $2.2 \pm 0.9-2.1 \pm 1.1 \mathrm{db}$. in groups 1 and 2, respectively, which was statistically similar ( $p>0.05)$ (Table 3).

Table 4 shows the correlations between SD-OCT measurements (average RGC-IPL and RNFL thickness), and standard automated 10-2 perimetry results (MD and sLV). MD and sLV were statistically similar. A statistically significant mild negative correlation was found between the cumulative dose of HCQ and average RGC-IPL thickness in group 1 (Figure 2a). Furthermore, the duration of drug use and average RGC-IPL thickness were also found to have a statistically

Table 3. Retinal nerve fiber layer thickness comparison between groups

\begin{tabular}{|c|c|c|c|}
\hline & Group 1 ( $n=92$ eyes) & Group 2 ( $\mathrm{n}=80$ eyes) & \\
\hline & Mean \pm SD & Mean \pm SD & $p^{*}$ \\
\hline \multicolumn{4}{|c|}{ RNFL thickness $(\mu \mathrm{m})$} \\
\hline Average** & $96.1 \pm 7.5$ & $96.6 \pm 11.5$ & 0.806 \\
\hline Superior & $119.6 \pm 18.9$ & $124 \pm 12.6$ & 0.113 \\
\hline Nasal & $64.2 \pm 8.3$ & $65 \pm 8.5$ & 0.991 \\
\hline Inferior & $127.4 \pm 19.3$ & $126.3 \pm 14.6$ & 0.724 \\
\hline Temporal & $75.3 \pm 13.7$ & $71.2 \pm 11.5$ & 0.083 \\
\hline
\end{tabular}

SD: Standard deviation; RNFL: Retinal nerve fiber layer; * Student-t test; ** Average value is calculated by taking arithmetic mean of other superior, inferior, nasal, and temporal thickness measurements.

Table 4. Correlations between measured thicknesses, automated perimetry parameters and cumulative dose and duration of hydroxychloroquine

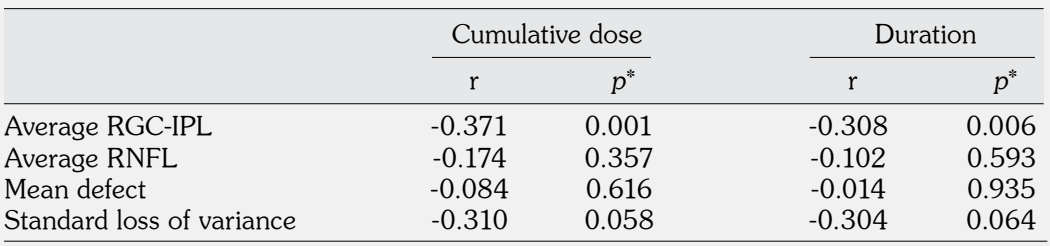

* Pearson's correlation test; RGC-IPL: Retinal ganglion cell-inner plexiform layer; RNFL: Retinal nerve fiber layer. 
(a)

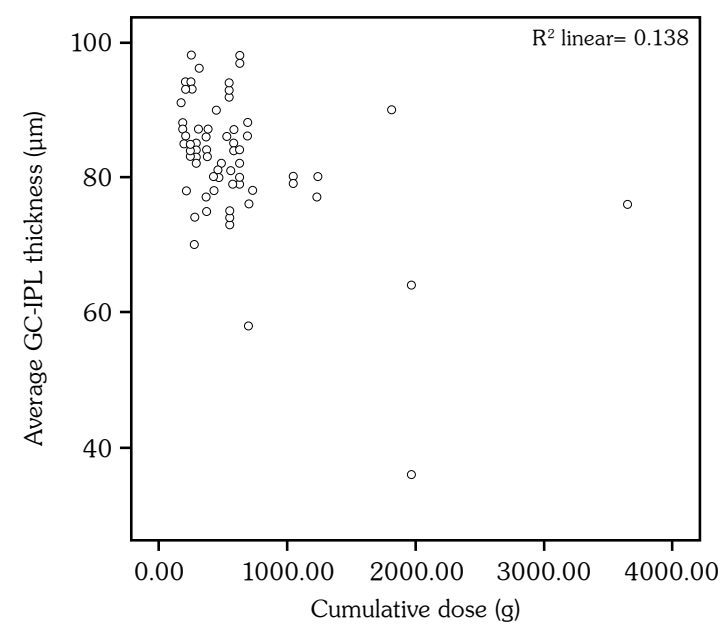

(b)

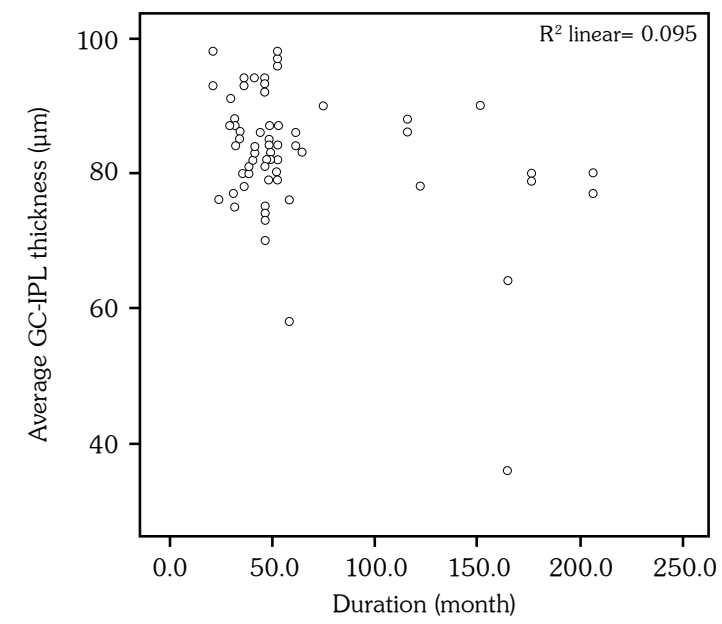

Figure 2. This figure shows correlation between average ganglion cell-inner plexiform layer (GC-IPL) thickness and cumulative dose of hydroxychloroquine (a), and duration of use of hydroxychloroquine (b).

significant mild negative correlation (Figure 2b). No statistically significant correlation was found between the average RNFL and the cumulative dose of the drug as well as the duration of use. Similarly, no statistically significant correlation was found between MD and the cumulative dose as well as the duration of use. sLV was found to have no statistically significant correlation with the cumulative dose and duration of use. Five out of 46 patients in group 1 were in high risk group for HCQ-induced retinopathy. Only one patient was diagnosed with clinically apparent HCQ retinopathy.

\section{DISCUSSION}

Retinopathy due to HCQ is associated with macular retinal pigmentary changes. The exact mechanism of HCQ-induced toxicity is not fully known. At earlier stages, drug accumulates in the cytoplasm of RGCs and, leads to cell shrinkage and formation of an irregular nucleus, which eventually results in ganglion cell degeneration. At later stages, it leads to the degeneration of photoreceptors and retinal pigment epithelium degeneration by binding on melanin ${ }^{9,10}$ and the resulting functional loss in visual field, decreased visual acuity and impairment of color vision. ${ }^{12}$ SD-OCT images revealed that patients taking HCQ suffered from the loss of the parafoveal photoreceptor inner segment-outer segment junction and thinning in their outer nuclear layer. ${ }^{13}$ Retinal thinning may be an early indicator of retinopathy. ${ }^{14}$

Hydroxychloroquine-induced retinal toxicity is a very rare condition. ${ }^{15}$ In a recent study, the rate of retinal toxicity was reported to be $0.65 \% \quad(n=3995)$. However, if the drug is used more than five years and the total cumulative dose exceeds 1000 grams, the toxicity rate exceeds $1 \% .{ }^{16}$ Drug dose of higher than $6.5 \mathrm{mg} / \mathrm{kg} /$ day (or $400 \mathrm{mg} /$ day), drug use for more than five years, and a cumulative dose higher than $1000 \mathrm{~g}$ are considered as high risk factors for HCQ-induced retinopathy. ${ }^{17}$ Toxicity can be evaluated by subjective tests such as visual acuity score, corneal slit lamp examination, fundus examination and automated central perimetry 10-2 test. ${ }^{4}$ Amsler grid testing, color vision testing, fluorescein angiography, electrooculogram and full-field ERG are no longer recommended as screening tests. ${ }^{17}$ Fundus autofluorescence photography, multifocal ERG and SD-OCT macular measurements are considered as objective tests. ${ }^{4}$ It is recommended to use at least one objective test in addition to the subjective tests for screening. ${ }^{18}$ In order to discontinue the treatment in patients who are suspected to have HCQ-induced retinal toxicity, it must be confirmed with at least two screening 
tests including minimum one objective screening test. ${ }^{19}$

Spectral-domain optical coherence tomography images show localized thinning of retinal layers in the parafoveal zone, and may reveal retinal toxicity at an earlier stage before visual field loss occurs. ${ }^{6,11,20-22}$ Majority of the previous studies were related to the pathologies associated with the outer retinal segment involving retinal pigment epithelium and photoreceptors. ${ }^{13,21,23}$ However, some other studies also demonstrated HCQassociated damage in the inner retinal segment. ${ }^{11,19}$ In our study, we also found statistically significant thinning of RGC-IPL, which is a part of the inner retina; average RGC-IPL thickness, and all segments except inferior segment in the group of patients who were taking HCQ compared to the controls who were not taking the drug. Moreover, the cumulative dose and duration use of HCQ were found to have a statistically significant negative correlation with average RGC-IPL thickness. The findings of our study are consistent with those reported in a similar study recently conducted by Lee et al. ${ }^{20}$

In our study, we did not find any difference between the groups with respect to RNFL thickness. This may be associated with the fact that thinning of RNFL is presented at later stages by the advanced degeneration of RGCs. In our study, we also did not find any difference between the groups with respect to the standard automated perimetry 10-2 results. Our findings demonstrated that HCQ-induced retinal toxicity could be detected at an earlier stage through the measurement of RGC-IPL thickness using SD-OCT before it appeared in visual field tests. Pasadhika et al. ${ }^{11}$ reported significantly reduced RGC-IPL thickness with similar findings of perimetry 10-2 results to our study in HCQ-treated patients. The patients included in our study had relatively short duration and lower cumulative dose of HCQ; in other words, had lower risk for HCQ-induced retinal toxicity. Nevertheless, the diminished thickness of retinal layer may be an antecedent finding of retinopathy.

Our study had two important limitations. The first limitation was the low number of patients included in the study; we suggest that it can be supported by further studies with higher number of subjects. The other limitation is the inability to compare the results with basal counterparts before the treatment. This may show the long-term effects of the drug. The HCQ was permitted to be in drug-markets for less than 10 years in our country. So, mean duration of drug's consumption is not so long in general. This is the main reason for the second limitation of our study. Further studies should be designed accordingly.

The follow-up of retinal toxicity induced by antimalarial drugs has been employed by various techniques: visual field analysis, fundus autofluorescence photography and ERG. However, these techniques do not allow detection of possible findings of retinal toxicity before full development. It is crucial to detect retinal toxicity induced by HCQ early and take necessary actions before it results in permanent visual loss. In our study, we demonstrated HCQ-induced retinal toxicity at an earlier stage through the measurement of RGC-IPL thickness by using SD-OCT, which is an easy-to-use and noninvasive method. Additionally, SD-OCT allows various evaluations on recorded retinal images. The clinicians primarily responsible for follow-up of antimalarial-induced side effects are advised to request $\mathrm{SD}-\mathrm{OCT}$ for evaluation of retinal toxicity from ophthalmology clinics.

In conclusion, we suggest that the measurement of RGC-IPL thickness may become an important objective in screening tests for HCQ-induced retinal toxicity. We need further studies, which should be performed with larger patient populations, to evaluate the retinotoxic effects of antimalarial drugs.

\section{Declaration of conflicting interests}

The authors declared no conflicts of interest with respect to the authorship and/or publication of this article.

\section{Funding}

The authors received no financial support for the research and/or authorship of this article.

\section{REFERENCES}

1. Yam JC, Kwok AK. Ocular toxicity of hydroxychloroquine. Hong Kong Med J 2006;12:294304. 
2. Rynes RI. Antimalarial drugs in the treatment of rheumatological diseases. $\mathrm{Br} \mathrm{J}$ Rheumatol 1997;36:799-805.

3. Korah S, Kuriakose T. Optical coherence tomography in a patient with chloroquine-induced maculopathy. Indian J Ophthalmol 2008;56:511-3.

4. Geamanu Panca A, Popa-Cherecheanu A, Marinescu B, Geamanu CD, Voinea LM. Retinal toxicity associated with chronic exposure to hydroxychloroquine and its ocular screening. Review. J Med Life 2014;7:322-6.

5. Mavrikakis I, Sfikakis PP, Mavrikakis E, Rougas K, Nikolaou A, Kostopoulos C, et al. The incidence of irreversible retinal toxicity in patients treated with hydroxychloroquine: a reappraisal. Ophthalmology 2003;110:1321-6.

6. Pasadhika S, Fishman GA. Effects of chronic exposure to hydroxychloroquine or chloroquine on inner retinal structures. Eye (Lond) 2010;24:340-6.

7. Çay HF, Erol MK, Çoban DT, Bulut M, Sezer I, Çakır T, et al. Retinotoxicity of hydroxychloroquine: is it possible to demonstrate by spectral domain optical coherence tomography before development? A cross sectional investigation. Arch Rheumatol 2014;29:178-85.

8. Wetterholm DH, Winter FC. Histopathology of chloroquine retinal toxicity. Arch Ophthalmol 1964;71:82-7.

9. Ramsey MS, Fine BS. Chloroquine toxicity in the human eye. Histopathologic observations by electron microscopy. Am J Ophthalmol 1972;73:229-35.

10. Rosenthal AR, Kolb H, Bergsma D, Huxsoll D, Hopkins JL. Chloroquine retinopathy in the rhesus monkey. Invest Ophthalmol Vis Sci 1978;17:1158-75.

11. Pasadhika S, Fishman GA, Choi D, Shahidi M. Selective thinning of the perifoveal inner retina as an early sign of hydroxychloroquine retinal toxicity. Eye (Lond) 2010;24:756-62.

12. Tehrani R, Ostrowski RA, Hariman R, Jay WM. Ocular toxicity of hydroxychloroquine. Semin Ophthalmol 2008;23:201-9.

13. Rodriguez-Padilla JA, Hedges TR, Monson B, Srinivasan V, Wojtkowski M, Reichel E, et al. High-speed ultra-high-resolution optical coherence tomography findings in hydroxychloroquine retinopathy. Arch Ophthalmol 2007;125:775-80.
14. Kellner S, Weinitz S, Kellner U. Spectral domain optical coherence tomography detects early stages of chloroquine retinopathy similar to multifocal electroretinography, fundus autofluorescence and near-infrared autofluorescence. $\mathrm{Br} \mathrm{J}$ Ophthalmol 2009;93:1444-7.

15. Levy GD, Munz SJ, Paschal J, Cohen HB, Pince $\mathrm{KJ}$, Peterson T. Incidence of hydroxychloroquine retinopathy in 1,207 patients in a large multicenter outpatient practice. Arthritis Rheum 1997;40:1482-6.

16. Wolfe F, Marmor MF. Rates and predictors of hydroxychloroquine retinal toxicity in patients with rheumatoid arthritis and systemic lupus erythematosus. Arthritis Care Res (Hoboken) 2010;62:775-84.

17. Marmor MF, Kellner U, Lai TY, Lyons JS, Mieler WF. Revised recommendations on screening for chloroquine and hydroxychloroquine retinopathy. Ophthalmology 2011;118:415-22.

18. Costedoat-Chalumeau N, Ingster-Moati I, Leroux G, Fardeau $\mathrm{C}$, Benveniste $\mathrm{O}$, Simon $\mathrm{C}$, et al. Critical review of the new recommendations on screening for hydroxychloroquine retinopathy. Rev Med Interne 2012;33:265-7. [Abstract]

19. Marmor MF. Efficient and effective screening for hydroxychloroquine toxicity. Am J Ophthalmol 2013;155:413-4.

20. Lee MG, Kim SJ, Ham DI, Kang SW, Kee C, Lee J, et al. Macular retinal ganglion cell-inner plexiform layer thickness in patients on hydroxychloroquine therapy. Invest Ophthalmol Vis Sci 2014;56:396-402.

21. de Sisternes L, Hu J, Rubin DL, Marmor MF. Localization of damage in progressive hydroxychloroquine retinopathy on and off the drug: inner versus outer retina, parafovea versus peripheral fovea. Invest Ophthalmol Vis Sci 2015;56:3415-26.

22. Stepien KE, Han DP, Schell J, Godara P, Rha J, Carroll J. Spectral-domain optical coherence tomography and adaptive optics may detect hydroxychloroquine retinal toxicity before symptomatic vision loss. Trans Am Ophthalmol Soc 2009;107:28-33.

23. Chen E, Brown DM, Benz MS, Fish RH, Wong TP, Kim RY, et al. Spectral domain optical coherence tomography as an effective screening test for hydroxychloroquine retinopathy (the "flying saucer" sign). Clin Ophthalmol 2010;4:1151-8. 